Article

\title{
Fuzzy Sumudu Transform for Solving System of Linear Fuzzy Differential Equations with Fuzzy Constant Coefficients
}

\author{
N.A. Abdul Rahman ${ }^{1, *}$ (1), M.Z. Ahmad ${ }^{2}$ \\ 1 School of Mathematical Sciences, Universiti Sains Malaysia, 11800 USM, Penang, Malaysia.; \\ aswad.rahman@usm.my \\ 2 Institute of Engineering Mathematics, Universiti Malaysia Perlis, Pauh Putra Main Campus, 02600 Arau, \\ Perlis, Malaysia; mzaini@unimap.edu.my \\ * Correspondence: aswad.rahman@usm.my; Tel.: +604-6533944
}

\begin{abstract}
In this paper, we employ fuzzy Sumudu transform for solving system of linear fuzzy differential equations with fuzzy constant coefficients. The system with fuzzy constant coefficients is interpreted under strongly generalized differentiability. For this purpose, new procedures for solving the system are proposed. A numerical example is carried out for solving system adapted from fuzzy radioactive decay model. Conclusion is drawn in the last section and some potential research directions are given.
\end{abstract}

Keywords: fuzzy Sumudu transform; fuzzy linear differential equations; system of fuzzy differential equations

MSC: 34A07, 65R10, 34A30

\section{Introduction}

For centuries, scientists have struggled to model real world phenomenon effectively and precisely. Among several modelling tools, scientists prefer to construct their models based on system of linear differential equations involving initial value problems [1-5]. This is because this kind of modelling are easier to be solved and analysed. However, the drawback of this modelling is very obvious and it is far from ideal. This is resulting from the lack of our knowledge about the world. Uncertainties or imprecisions occur in almost every aspect in our life, especially when dealing with real life phenomenon such as microbes, air and population. Using ordinary differential equations, the uncertainties are not dealt accordingly, thus, this leads to an inexact model.

To handle this shortcoming, scientist make use of Zadeh fuzzy set theory where the author emphasized that a number can be classified into certain membership function rather than we represent it as a discrete or crisp number [6]. The new modelling tool is referred to as fuzzy differential equations (FDEs). FDEs are developed whenever the structure of the model is non probabilistic. Unlike ordinary differential equations, FDEs take accounts the uncertainties or imprecisions observed circulating around the problem. There are a vast literature that can be found discussing FDEs as well as fuzzy derivatives [7-12].

So, the effort to construct methods for solving FDEs become an urgent matter. This includes both analytical and numerical methods. However, before we develop numerical methods for dealing with FDEs, it is important for us to construct analytical methods.

Recently, Ahmad and Abdul Rahman [13,14], proposed fuzzy Sumudu transform (FST) for solving FDEs with fuzzy initial values which was done under the strongly generalized differentiability concept. The authors successfully introduced some of FST fundamental properties and theories, and later demonstrated the proposed method on several numerical examples. As stated by the authors, the advantage of FST is significant as it reduces the complexity of the calculation when handling FDEs. 
Plus, the final unit of the solution can be seen without even completing the solving process. This is due to its scale preserving property. Then, FST is used to solve fuzzy partial differential equations [15] before later, Haydar used FST to solve nth-order FDEs with fuzzy initial values [16]. The studies are the followed by the application of FST on fuzzy fractional differential equations and fuzzy Volterra integral equations in [17] and [18] respectively.

Scientists had continue utilizing FDEs to construct a more complex model which consists of several FDEs. The stepping stone for this effort is the development of system of linear first order fuzzy differential equations (SLFDEs). There has been several works regarding SLFDEs with fuzzy constant coefficients (FCCs) in the literature [19-24]. These include the implementation of variational iteration method and homotopy analysis method. When dealing with FDEs interpreted under strongly generalized differentiability concept, there are two cases of differentiability to be considered [11]. The previous works done did not demonstrated both cases efficiently, for example, the work in [19]. Particularly, only the first case is demonstrated by the authors. Because of this, we intend to use FST for solving SLFDEs with FCCs, and both cases of differentiability interpreted under the mentioned concept will be fully demonstrated. Plus, to the best of our knowledge, this is the first time FST is used to handle such system.

This paper is organized as follows. In Section 2, we recall several basic definitions and concepts of fuzzy numbers and fuzzy derivatives. In Section 3, we provide a general definition of FST. In addition, we also discuss on the scale preserving property possessed by FST and a brief comparison was done with fuzzy Laplace transform which has been proposed in [25]. Next in Section 4, we provide some details on the SLFDEs where two cases of the strongly generalized differentiability are considered. In Section 5, we construct detailed procedures to solve SLFDEs with FCCs. While in Section 6, a numerical example is demonstrated to show that the proposed method is practical. Later, in Section 7 , we give conclusions.

\section{Preliminaries}

Several important definitions and properties for fuzzy numbers and fuzzy functions are recalled in this section. Note that the real number and fuzzy number are denoted by $\mathbb{R}$ and $\mathcal{F}(\mathbb{R})$, respectively, throughout this paper.

Definition 1. [6] A fuzzy number is a mapping $\widetilde{u}: \mathbb{R} \rightarrow[0,1]$ that satisfies the following conditions.

1. For every $\widetilde{u} \in \mathcal{F}(\mathbb{R}), \widetilde{u}$ is upper semi continuous,

2. for every $\widetilde{u} \in \mathcal{F}(\mathbb{R}), \widetilde{u}$ is fuzzy convex, i.e., $\widetilde{u}(\gamma x+(1-\gamma) y) \geq \min \{\widetilde{u}(x), \widetilde{u}(y)\}$ for all $x, y \in \mathbb{R}$, and $\gamma \in[0,1]$

3. for every $\widetilde{u} \in \mathcal{F}(\mathbb{R}), \widetilde{u}$ is normal, i.e., $\exists x_{0} \in \mathbb{R}$ for which $\widetilde{u}\left(x_{0}\right)=1$,

4. supp $\widetilde{u}=\{x \in \mathbb{R} \mid \widetilde{u}(x)>0\}$ is the support of $\widetilde{u}$, and it has a compact closure cl(supp $\widetilde{u}$ ).

Definition 2. [26] Let $\widetilde{u} \in \mathcal{F}(\mathbb{R})$ and $\alpha \in] 0,1]$. The $\alpha$-level set of $\widetilde{u}$ is the crisp set $\widetilde{u}^{\alpha}$ that contains all the elements with membership degree greater than or equal to $\alpha$, i.e.

$$
\widetilde{u}^{\alpha}=\{x \in \mathbb{R} \mid \widetilde{u}(x) \geq \alpha\},
$$

where $\widetilde{u}^{\alpha}$ denotes $\alpha$-level set of fuzzy number $\widetilde{u}$.

It can be stated that the $\alpha$-level of any fuzzy number is a bounded and closed set, and it is denoted by $\left[\underline{u}^{\alpha}, \bar{u}^{\alpha}\right]$, for both lower and upper bound of $\widetilde{u}^{\alpha}$, respectively.

Definition 3. [10,27] A parametric form of an arbitrary fuzzy number $\widetilde{u}$ is an ordered pair $\left[\underline{u}^{\alpha}, \bar{u}^{\alpha}\right]$ of functions $\underline{u}^{\alpha}$ and $\bar{u}^{\alpha}$, for any $\alpha \in[0,1]$, that fulfil the following conditions.

1. $\underline{u}^{\alpha}$ is a bounded left continuous monotonic increasing function in $[0,1]$, 
2. $\bar{u}^{\alpha}$ is a bounded left continuous monotonic decreasing function in $[0,1]$,

3. $\underline{u}^{\alpha} \leq \bar{u}^{\alpha}$.

A fuzzy number is classified into certain membership function. In the literature, there are many types of membership function defined by researchers. For example, triangular, trapezoidal, Gaussian and generalized bell membership function. Among all of them, the triangular membership function is the most widely utilised by researchers. It is represented by three crisp numbers $\left(a_{1}, a_{2}, a_{3}\right)$ and its $\alpha$-level is computed as below [28].

$$
\widetilde{u}^{\alpha}=\left[a_{1}+\left(a_{2}-a_{3}\right) \alpha, a_{3}-\left(a_{3}-a_{2}\right) \alpha\right], \quad \alpha \in[0,1] .
$$

Basic operations between fuzzy numbers can be seen in [27].

Theorem 1. [29] Let the fuzzy function $\widetilde{f}: \mathbb{R} \rightarrow \mathcal{F}(\mathbb{R})$ represented by $\left[f^{\alpha}(x), \bar{f}^{\alpha}(x)\right]$. For any $\alpha \in[0,1]$, assume that $f^{\alpha}(x)$ and $\bar{f}^{\alpha}(x)$ are both Riemann-integrable on $[a, b]$ and assume that there are two positive $\underline{M}^{\alpha}$ and $\bar{M}^{\alpha}$ where $\int_{a}^{b}\left|f^{\alpha}(x)\right| d x \leq \underline{M}^{\alpha}$ and $\int_{a}^{b}\left|\bar{f}^{\alpha}(x)\right| d x \leq \bar{M}^{\alpha}$, for every $b \geq a$. Then, $\tilde{f}(x)$ is improper fuzzy Riemann-integrable on $[a, \infty[$ and the improper fuzzy Riemann-integrable is a fuzzy number. Furthermore, we have

$$
\int_{a}^{\infty} \widetilde{f}(x) d x=\left[\int_{a}^{\infty} \underline{f}^{\alpha}(x) d x, \int_{a}^{\infty} \bar{f}^{\alpha}(x) d x\right] .
$$

Definition 4. [9] If $\widetilde{u}, \widetilde{v} \in \mathcal{F}(\mathbb{R})$ and if there exists a fuzzy subset $\xi \in \mathcal{F}(\mathbb{R})$ such that $\xi+\widetilde{u}=\widetilde{v}$, then $\xi$ is unique. In this case, $\xi$ is called the Hukuhara difference, or simply H-difference, of $u$ and $v$ and is denoted by $\widetilde{v}-H \tilde{u}$. definition.

The strongly generalized differentiability concept for a fuzzy function is described in the following

Definition 5. [12,30] Let $\tilde{f}:] a, b\left[\rightarrow \mathcal{F}(\mathbb{R})\right.$ be a fuzzy function and $\left.x_{0} \in\right] a, b[$. We say that $\tilde{f}$ is strongly generalized differentiable on $x_{0}$, if there exists an element $\widetilde{f}^{\prime}\left(x_{0}\right) \in \mathcal{F}(\mathbb{R})$, such that

1. for all $h>0$ sufficiently small, $\exists \widetilde{f}\left(x_{0}+h\right)-{ }^{H} \widetilde{f}\left(x_{0}\right), \widetilde{f}\left(x_{0}\right)-{ }^{H} \widetilde{f}\left(x_{0}-h\right)$ and the limits (in the metric D)

$$
\begin{aligned}
\lim _{h \rightarrow 0} \frac{\tilde{f}\left(x_{0}+h\right)-{ }^{H} \tilde{f}\left(x_{0}\right)}{h} & =\lim _{h \rightarrow 0} \frac{\tilde{f}\left(x_{0}\right)-{ }^{H} \tilde{f}\left(x_{0}-h\right)}{h} \\
& =\widetilde{f}^{\prime}\left(x_{0}\right),
\end{aligned}
$$

or

2. for all $h>0$ sufficiently small, $\exists \widetilde{f}\left(x_{0}\right)-{ }^{H} \widetilde{f}\left(x_{0}+h\right), \widetilde{f}\left(x_{0}-h\right)-{ }^{H} \widetilde{f}\left(x_{0}\right)$ and the limits (in the metric D)

$$
\begin{aligned}
\lim _{h \rightarrow 0} \frac{\tilde{f}\left(x_{0}\right)-{ }^{H} \tilde{f}\left(x_{0}+h\right)}{-h} & =\lim _{\substack{h \rightarrow 0\\
}} \frac{\widetilde{f}\left(x_{0}-h\right)-{ }^{H} \tilde{f}\left(x_{0}\right)}{-h} \\
& =\widetilde{f}^{\prime}\left(x_{0}\right) .
\end{aligned}
$$

In this paper, the first part of the Definition 5 is referred to as (i)-differentiable, while the second part as (ii)-differentiable. 
Definition 6. [31] A fuzzy function $\tilde{f}:[a, b] \rightarrow \mathcal{F}(\mathbb{R})$ is said to be continuous at $x_{0} \in[a, b]$ if for each $\epsilon>0$, there is $\delta>0$ such that $D\left(\widetilde{f}(x), \widetilde{f}\left(x_{0}\right)\right)<\epsilon$, whenever $x \in[a, b]$ and $\left|x-x_{0}\right|<\delta$. We say that $\widetilde{f}$ is continuous on $[a, b]$ if $\tilde{f}$ is continuous at each $x_{0} \in[a, b]$.

Theorem 2. [11] Let $\widetilde{f}: \mathbb{R} \rightarrow \mathcal{F}(\mathbb{R})$ be a continuous fuzzy function and $\widetilde{f}(x)=\left[f^{\alpha}(x), \bar{f}^{\alpha}(x)\right]$, for every $\alpha \in[0,1]$. Then

1. if the fuzzy function $\widetilde{f}$ is (i)-differentiable, then $\underline{f}^{\alpha}(x)$ and $\bar{f}^{\alpha}(x)$ are both differentiable and

$$
f^{\prime}(x)=\left[\left(\underline{f}^{\prime}\right)^{\alpha}(x),\left(\bar{f}^{\prime}\right)^{\alpha}(x)\right],
$$

2. if the fuzzy function $\tilde{f}$ is (ii)-differentiable, then $\underline{f}^{\alpha}(x)$ and $\bar{f}^{\alpha}(x)$ are both differentiable and

$$
f^{\prime}(x)=\left[\left(\bar{f}^{\prime}\right)^{\alpha}(x),\left(f^{\prime}\right)^{\alpha}(x)\right] .
$$

The next section provides the previously constructed FST. The concept for SLFDEs under the strongly generalized differentiability concept is also provided in this section.

\section{Fuzzy Sumudu Transform}

In order to construct the solution for SLFDEs, we adopted the Definition of FST introduced in [14].

Definition 7. [14] Let $\widetilde{f}: \mathbb{R} \rightarrow \mathcal{F}(\mathbb{R})$ be a continuous fuzzy function. Suppose that $\widetilde{f}(u x) \odot e^{-x}$ is improper fuzzy Riemann-integrable on $\left[0, \infty\left[\right.\right.$, then $\int_{0}^{\infty} \widetilde{f}(u x) \odot e^{-x} d x$ is called fuzzy Sumudu transform and is denoted by

$$
G(u)=\mathcal{S}[\widetilde{f}(x)](u)=\int_{0}^{\infty} \widetilde{f}(u x) \odot e^{-x} d x, \quad u \in\left[-\tau_{1}, \tau_{2}\right],
$$

where the variable $u$ is used to factor the variable $x$ in the argument of the fuzzy function and $\tau_{1}, \tau_{2}>0$.

FST can also be parametrically written as follows.

$$
\mathcal{S}[\widetilde{f}(x)](u)=\left[s\left[\underline{f}^{\alpha}(x)\right](u), s\left[\bar{f}^{\alpha}(x)\right](u)\right] .
$$

To explore theorems of FST, please see in $[13,14]$. In the following subsection, we provide a brief discussion on the scale preserving property of FST and for the sake of comparison, we introduce the time scaling property of fuzzy Laplace Transform. This is the first time such property of fuzzy Laplace transform is introduced in the literature, and it is mainly to compare how scaling effects both FST and fuzzy Laplace transform.

\subsection{Discussion on Scale Preserving Property}

One of the most frequently highlighted property of FST is the scale preserving property. The theorems for scale preserving are as follows.

Theorem 3. [14](First preserving Theorem) Let $f: \mathbb{R} \rightarrow \mathcal{F}(\mathbb{R})$ be a continuous fuzzy-valued function and $a$ is an arbitrary constant, then

$$
\mathcal{S}[f(a t)]=G(a u) .
$$

Theorem 4. [14] (Second preserving Theorem) Let $f: \mathbb{R} \rightarrow \mathcal{F}(\mathbb{R})$ be a continuous fuzzy-valued function, then

$$
\mathcal{S}\left[t \odot \frac{d f(t)}{d t}\right]=u \frac{d G(u)}{d u} .
$$


The theorems listed are the first and second preserving Theorem, respectively. From the scale preserving Theorems, it can be said that FST may be used to solve problems without resorting to a new frequency domain. Unlike other integral transforms, the integral transforms need to be resorted to a new domain before they can be solved. For example, fuzzy Laplace transform, where its time scaling is given by the following theorem.

Theorem 5. Let $f: \mathbb{R} \rightarrow \mathcal{F}(\mathbb{R})$ be a continuous fuzzy-valued function and $a$ is an arbitrary constant, then

$$
\mathcal{L}[f(a t)]=\frac{1}{|a|} F\left(\frac{s}{a}\right),
$$

where $\mathcal{L}$ is the fuzzy Laplace transform introduced in [25].

Proof. From [25], the parametric form of fuzzy Laplace transform is given as follows.

$$
\begin{aligned}
\mathcal{L}[f(a t)] & =[l[f(a t)], l[f(a t)]], \\
& =\left[\int_{0}^{\infty} \underline{f}_{\alpha}(a t) e^{-s t} d t, \int_{0}^{\infty} \bar{f}_{\alpha}(a t) e^{-s t} d t\right] .
\end{aligned}
$$

We let $m=a t$, so, $\frac{d m}{a}=d t$. Then, we have

$$
\begin{aligned}
\mathcal{L}[f(a t)] & =\left[\int_{0}^{\infty} \underline{f}_{\alpha}(m) e^{-s(m / a)} \frac{d m}{a}, \int_{0}^{\infty} \bar{f}_{\alpha}(m) e^{-s(m / a)} \frac{d m}{a}\right], \\
& =\frac{1}{a}\left[\int_{0}^{\infty} \underline{f}_{\alpha}(m) e^{-m(s / a)} d m, \int_{0}^{\infty} \bar{f}_{\alpha}(m) e^{-m(s / a)} d m\right] .
\end{aligned}
$$

By the definition of fuzzy Laplace transform, finally,

$$
\mathcal{L}[f(a t)]=\frac{1}{a} F\left(\frac{s}{a}\right) .
$$

From Theorems 3,4 and 5, we can see that, the scale is preserved when we apply FST to the original function but not when we apply fuzzy Laplace transform. This property of FST made it possible for researchers to treat the transformed function as a replica to the original function. In addition, this property also allows researchers to obtain the unit of the final solution even without transforming it back to the original domain. Such virtue is important for validation of the final results when complex systems are encountered.

\section{System of First Order Linear Fuzzy Differential Equations}

In this part, we consider the following linear system that has been presented in several papers previously [11,26,32]. Based on the papers, the FDEs are in a system of ordinary differential equations. Additionally, in this paper, the system are extended to two cases, in the sense of differentiability as in Definition 5.

$$
\widetilde{X}^{\prime}(t)=\widetilde{A} \odot \widetilde{X}(t) \oplus \widetilde{f}(t)
$$

subjects to the initial conditions

$$
\widetilde{X}(0)=\widetilde{X}_{0}
$$

where the constant coefficients are $A, \widetilde{X}(t)=\left[X_{1}(t), X_{2}(t), \ldots, X_{n}(t)\right]^{T}, \widetilde{f}(t)=\left[f_{1}(t), f_{2}(t), \ldots, f_{n}(t)\right]^{T}$, $\widetilde{X}_{0}=\left[X_{10}, X_{20}, \ldots, X_{n 0}\right]$ and the elements of the vector $\widetilde{f}(t)$ are all fuzzy numbers. This means that the system consists of FDEs with FCCs. 
Let $\left[X_{m}(t)\right]^{\alpha}=\left[\left(\underline{X}_{m}\right)^{\alpha}(t),\left(\bar{X}_{m}\right)^{\alpha}(t)\right], m=1,2, \ldots, n$, if $X_{m}(t)$ is (i)-differentiable, then $\left[X_{m}^{\prime}(t)\right]^{\alpha}=$ $\left[\left(\underline{X}_{m}^{\prime}\right)^{\alpha}(t),\left(\bar{X}_{m}^{\prime}\right)^{\alpha}(t)\right]$. This is resulting from Theorem 2 . Thus for $m=1,2, \ldots, n$, we have

$$
\begin{array}{r}
\left(\underline{X}_{m}^{\prime}\right)^{\alpha}(t)=\sum_{j=1}^{n} \frac{a_{m j}^{\alpha}\left(X_{j}\right)^{\alpha}(t)}{\left(\underline{X}_{m}\right)^{\alpha}(0)}+\left(\underline{f}_{m}\right)^{\alpha}(t), \\
\left(\underline{X}_{m 0}\right)^{\alpha}, \\
\bar{X}^{\alpha}(t)=\sum_{j=1}^{n} \frac{a_{m j}^{\alpha}\left(X_{j}\right)^{\alpha}(t)}{a_{m}}+\left(\bar{f}_{m}\right)^{\alpha}(t), \\
\left(\bar{X}_{m}\right)^{\alpha}(0)=\left(\bar{X}_{m 0}\right)^{\alpha},
\end{array}
$$

and if $X_{m}(t)$ is (ii)-differentiable, we have $\left[X_{m}^{\prime}(t)\right]^{\alpha}=\left[\left(\bar{X}_{m}^{\prime}\right)^{\alpha}(t),\left(\underline{X}_{m}^{\prime}\right)^{\alpha}(t)\right]$. So,

$$
\begin{array}{r}
\left(\bar{X}_{m}^{\prime}\right)^{\alpha}(t)=\sum_{j=1}^{n} \frac{a_{m j}^{\alpha}\left(X_{j}\right)^{\alpha}(t)}{+}+\left(\underline{f}_{m}\right)^{\alpha}(t), \\
\left(\bar{X}_{m}\right)^{\alpha}(0)=\left(\bar{X}_{m 0}\right)^{\alpha}, \\
\underline{X}_{m}^{\prime}(t)=\sum_{j=1}^{n} \frac{a_{m j}^{\alpha}\left(X_{j}\right)^{\alpha}(t)}{}+\left(\bar{f}_{m}\right)^{\alpha}(t), \\
\left(\underline{X}_{m}\right)^{\alpha}(0)=\left(\underline{X}_{m 0}\right)^{\alpha},
\end{array}
$$

where

$$
\begin{aligned}
& \frac{a_{m j}^{\alpha}\left(X_{j}\right)^{\alpha}(t)}{\overline{a_{m j}^{\alpha}\left(X_{j}\right)^{\alpha}(t)}}=\min \left\{A X \mid A \in\left[\left(\underline{a}_{m j}\right)^{\alpha},\left(\bar{a}_{m j}\right)^{\alpha}\right], X \in\left[\left(\underline{X}_{j}\right)^{\alpha}(t),\left(\bar{X}_{j}\right)^{\alpha}(t)\right]\right\}, \\
& \max \left\{A X \mid A \in\left[\left(\underline{a}_{m j}\right)^{\alpha},\left(\bar{a}_{m j}\right)^{\alpha}\right], X \in\left[\left(\underline{X}_{j}\right)^{\alpha}(t),\left(\bar{X}_{j}\right)^{\alpha}(t)\right]\right\} .
\end{aligned}
$$

Consequently, if $X_{m}(t)$ is (i)-differentiable, Eq. (4) is interpreted as the following.

1. If $a_{m j}$ is non negative,

$$
\begin{array}{r}
\left(\underline{X}_{m}^{\prime}\right)^{\alpha}(t)=\sum_{j=1}^{n}\left(\underline{a}_{m j}\right)^{\alpha}\left(\underline{X}_{j}\right)^{\alpha}(t)+\left(\underline{f}_{m}\right)^{\alpha}(t), \\
\left(\underline{X}_{m}\right)^{\alpha}(0)=\left(\underline{X}_{m 0}\right)^{\alpha}, \\
\left(\bar{X}_{m}^{\prime}\right)^{\alpha}(t)=\sum_{j=1}^{n}\left(\bar{a}_{m j}\right)^{\alpha}\left(\bar{X}_{j}\right)^{\alpha}(t)+\left(\bar{f}_{m}\right)^{\alpha}(t), \\
\left(\bar{X}_{m}\right)^{\alpha}(0)=\left(\bar{X}_{m 0}\right)^{\alpha},
\end{array}
$$

2. if $a_{m j}$ is non positive,

$$
\begin{array}{r}
\left(\underline{X}_{m}^{\prime}\right)^{\alpha}(t)=\sum_{j=1}^{n}\left(\underline{a}_{m j}\right)^{\alpha}\left(\bar{X}_{j}\right)^{\alpha}(t)+\left(\underline{f}_{m}\right)^{\alpha}(t), \\
\left(\underline{X}_{m}\right)^{\alpha}(0)=\left(\underline{X}_{m 0}\right)^{\alpha}, \\
\left(\bar{X}_{m}^{\prime}\right)^{\alpha}(t)=\sum_{j=1}^{n}\left(\bar{a}_{m j}\right)^{\alpha}\left(\underline{X}_{j}\right)^{\alpha}(t)+\left(\bar{f}_{m}\right)^{\alpha}(t), \\
\left(\bar{X}_{m}\right)^{\alpha}(0)=\left(\bar{X}_{m 0}\right)^{\alpha} .
\end{array}
$$

And if $X_{m}(t)$ is (ii)-differentiable, Eq. (5) is interpreted as follows. 
1. If $a_{m j}$ is non negative,

$$
\begin{array}{r}
\left(\bar{X}_{m}^{\prime}\right)^{\alpha}(t)=\sum_{j=1}^{n}\left(\underline{a}_{m j}\right)^{\alpha}\left(\underline{X}_{j}\right)^{\alpha}(t)+\left(\underline{f}_{m}\right)^{\alpha}(t), \\
\left(\underline{X}_{m}\right)^{\alpha}(0)=\left(\underline{X}_{m 0}\right)^{\alpha}, \\
\left(\underline{X}_{m}^{\prime}\right)^{\alpha}(t)=\sum_{j=1}^{n}\left(\bar{a}_{m j}\right)^{\alpha}\left(\bar{X}_{j}\right)^{\alpha}(t)+\left(\bar{f}_{m}\right)^{\alpha}(t), \\
\left(\bar{X}_{m}\right)^{\alpha}(0)=\left(\bar{X}_{m 0}\right)^{\alpha},
\end{array}
$$

2. if $a_{m j}$ is non positive,

$$
\begin{array}{r}
\left(\bar{X}_{m}^{\prime}\right)^{\alpha}(t)=\sum_{j=1}^{n}\left(\underline{a}_{m j}\right)^{\alpha}\left(\bar{X}_{j}\right)^{\alpha}(t)+\left(\underline{f}_{m}\right)^{\alpha}(t), \\
\left(\underline{X}_{m}\right)^{\alpha}(0)=\left(\underline{X}_{m 0}\right)^{\alpha}, \\
\left(\underline{X}_{m}^{\prime}\right)^{\alpha}(t)=\sum_{j=1}^{n}\left(\bar{a}_{m j}\right)^{\alpha}\left(\underline{X}_{j}\right)^{\alpha}(t)+\left(\bar{f}_{m}\right)^{\alpha}(t), \\
\left(\bar{X}_{m}\right)^{\alpha}(0)=\left(\bar{X}_{m 0}\right)^{\alpha} .
\end{array}
$$

5. Fuzzy Sumudu Transform for System of Linear First Order Fuzzy Differential Equations

Consider the following SLFDEs [26].

$$
\widetilde{X}^{\prime}(t)=\widetilde{A} \odot \widetilde{X}(t) \oplus \widetilde{f}(t),
$$

subjects to the initial conditions

$$
\widetilde{X}(0)=\widetilde{X}_{0}
$$

Eq. (11) can also be rewritten as

$$
\begin{gathered}
\widetilde{X}_{1}^{\prime}=\sum_{j=1}^{n} \widetilde{a}_{1 j} \widetilde{X}_{j}(t)+\widetilde{f}_{1}(t), \\
\widetilde{X}_{2}^{\prime}=\sum_{j=1}^{n} \widetilde{a}_{2 j} \widetilde{X}_{j}(t)+\widetilde{f}_{2}(t), \\
\vdots \\
\widetilde{X}_{n}^{\prime}=\sum_{j=1}^{n} \widetilde{a}_{n j} \widetilde{X}_{j}(t)+\widetilde{f}_{n}(t),
\end{gathered}
$$

or simply,

$$
\widetilde{X}_{m}^{\prime}=\sum_{j=1}^{n} \widetilde{a}_{m j} \widetilde{X}_{j}(t)+\widetilde{f}_{m}(t),
$$

for $m=1,2, \ldots, n$, subjects to initial conditions $\widetilde{X}_{0}=\left[X_{10}, X_{20}, \ldots, X_{n 0}\right]$. By using FST on both sides of Eq. (14), we have

$$
\mathcal{S}\left[\widetilde{X}_{m}^{\prime}\right](u)=\mathcal{S}\left[\sum_{j=1}^{n} \widetilde{a}_{m j} \widetilde{X}_{j}(t)+\widetilde{f}_{m}(t)\right](u) .
$$

From Theorem 2, we know that the conditions of the fuzzy derivative happened by two cases, which are (i)-differentiable or (ii)-differentiable. So, for $m=1,2, \ldots, n$, if $\widetilde{X}_{m}$ is (i)-differentiable, we 
have $\widetilde{X}_{m}^{\prime}=\left[\underline{X}_{m}^{\prime}, \bar{X}_{m}^{\prime}\right]$. If $\widetilde{X}_{m}$ is (ii)-differentiable, we have $\widetilde{X}_{m}^{\prime}=\left[\bar{X}_{m}^{\prime}, \underline{X}_{m}^{\prime}\right]$.

By Theorem 3.1 in [13], if $\widetilde{X}_{m}$ is (i)-differentiable, we have

$$
\mathcal{S}\left[\widetilde{X}_{m}^{\prime}\right](u)=\frac{G(u)-{ }^{H} \widetilde{X}_{m}(0)}{u},
$$

and this means that,

$$
\begin{aligned}
& s\left[\sum_{j=1}^{n} a_{m j}^{\alpha}\left(X_{j}\right)^{\alpha}(t)+\left(\underline{f}_{m}\right)^{\alpha}(t)\right](u)=\frac{s\left[\left(\underline{X}_{m}\right)^{\alpha}(t)\right](u)-\left(\underline{X}_{m}\right)^{\alpha}(0)}{u}, \\
& s\left[\sum_{j=1}^{n} \overline{a_{m j}^{\alpha}\left(X_{j}\right)^{\alpha}(t)}+\left(\bar{f}_{m}\right)^{\alpha}(t)\right](u)=\frac{s\left[\left(\bar{X}_{m}\right)^{\alpha}(t)\right](u)-\left(\bar{X}_{m}\right)^{\alpha}(0)}{u},
\end{aligned}
$$

where $m=1,2, \ldots, n$.

Also by Theorem 3.1 in [13], if $\widetilde{X}_{m}$ is (ii)-differentiable, we have

$$
\mathcal{S}\left[\widetilde{X}_{m}\right](u)=\frac{-\widetilde{X}_{m}(0)-{ }^{H}(-G(u))}{u},
$$

and this means that,

$$
\begin{aligned}
& s\left[\sum_{j=1}^{n} a_{m j}^{\alpha}\left(X_{j}\right)^{\alpha}(t)+\left(\underline{f}_{m}\right)^{\alpha}(t)\right](u)=\frac{s\left[\left(\bar{X}_{m}\right)^{\alpha}(t)\right](u)-\left(\bar{X}_{m}\right)^{\alpha}(0)}{u}, \\
& s\left[\sum_{j=1}^{n} \overline{a_{m j}^{\alpha}\left(X_{j}\right)^{\alpha}(t)}+\left(\bar{f}_{m}\right)^{\alpha}(t)\right](u)=\frac{s\left[\left(\underline{X}_{m}\right)^{\alpha}(t)\right](u)-\left(\underline{X}_{m}\right)^{\alpha}(0)}{u},
\end{aligned}
$$

where $m=1,2, \ldots, n$.

To solve Eq. (16) or (17), we assume that

$$
\begin{aligned}
& s\left[\left(\underline{X}_{m}\right)^{\alpha}(t)\right](u)=L_{m}^{\alpha}(u), \\
& s\left[\left(\bar{X}_{m}\right)^{\alpha}(t)\right](u)=U_{m}^{\alpha}(u),
\end{aligned}
$$

where $L_{1}^{\alpha}(u), U_{1}^{\alpha}(u), L_{2}^{\alpha}(u), U_{2}^{\alpha}(u), \ldots, L_{n}^{\alpha}(u)$ and $U_{n}^{\alpha}(u)$ are the solutions of Eq. (16) or (17). Then, by the inverse of FST, the final solutions are as the following.

$$
\begin{aligned}
& \left(\underline{X}_{m}\right)^{\alpha}(t)=s^{-1}\left[L_{m}^{\alpha}(u)\right], \\
& \left(\bar{X}_{m}\right)^{\alpha}(t)=s^{-1}\left[U_{m}^{\alpha}(u)\right] .
\end{aligned}
$$

\section{Numerical Example}

The developed procedures is then applied on a numerical example. This is meant to demonstrate the applicability of FST in solving SLFDEs with FCCs.

Example 1. The following model is adapted from fuzzy radioactive decay model from [20]. In this model, $r, N_{1}$, $N_{2}, \widetilde{\lambda}_{1}$ and $\widetilde{\lambda}_{2}$ are considered to be triangular fuzzy numbers.

$$
\begin{aligned}
& N_{1}^{\prime}(t)=-\widetilde{\lambda}_{1} N_{1}(t)+r, \\
& N_{2}^{\prime}(t)=\widetilde{\lambda}_{1} N_{1}(t)-\widetilde{\lambda}_{2} N_{2}(t), \\
& N_{1}(0)=N_{1}, \quad N_{2}(0)=N_{2} .
\end{aligned}
$$


In [20], the authors stated that the values of $r, N_{1}, N_{2}, \widetilde{\lambda}_{1}$ and $\widetilde{\lambda}_{2}$ may be acquired from experts. The lowest, the highest and the most exact values for $r, N_{1}, N_{2}$ and $\widetilde{\lambda}_{1}$ and $\widetilde{\lambda}_{2}$ can be considered, and from this, five triangular fuzzy numbers are constructed. Their corresponding parametric representations can be calculated using Eq. (1). By using FST on both sides of Eq. (20),

$$
\begin{aligned}
& \mathbb{S}\left[N_{1}^{\prime}(t)\right](u)=\mathbb{S}\left[-\lambda_{1} N_{1}(t)+r\right](u) \\
& \mathbb{S}\left[N_{2}^{\prime}(t)\right](u)=\mathbb{S}\left[\lambda_{1} N_{1}(t)-\lambda_{2} N_{2}(t)\right](u)
\end{aligned}
$$

This is equivalent to

$$
\begin{aligned}
& \mathbb{S}\left[N_{1}^{\prime}(t)\right](u)=-\lambda_{1} \mathbb{S}\left[N_{1}(t)\right](u)+r \\
& \mathbb{S}\left[N_{2}^{\prime}(t)\right](u)=\lambda_{1} \mathbb{S}\left[N_{1}(t)\right](u)-\lambda_{2} \mathbb{S}\left[N_{2}(t)\right](u) .
\end{aligned}
$$

Then, the procedures for solving Eq. (22) are divided into two cases.

Case 1: First, consider both $N_{1}(t)$ and $N_{2}(t)$ to be (i)-differentiable. Then,

$$
\begin{aligned}
& \frac{s\left[\underline{N}_{1}(t)\right](u)-\underline{N}_{1}(0)}{u}=-\bar{\lambda}_{1} s\left[\bar{N}_{1}(t)\right](u)+\underline{r}, \\
& \frac{s\left[\bar{N}_{1}(t)\right](u)-\bar{N}_{1}(0)}{u}=-\underline{\lambda}_{1} s\left[\underline{N}_{1}(t)\right](u)+\bar{r}, \\
& \frac{s\left[\underline{N}_{2}(t)\right](u)-\underline{N}_{2}(0)}{u}=\underline{\lambda}_{1} s\left[\underline{N}_{1}(t)\right](u)-\bar{\lambda}_{2} s\left[\bar{N}_{2}(t)\right](u), \\
& \frac{s\left[\bar{N}_{2}(t)\right](u)-\bar{N}_{2}(0)}{u}=\bar{\lambda}_{1} s\left[\bar{N}_{1}(t)\right](u)-\underline{\lambda}_{2} s\left[\underline{N}_{2}(t)\right](u) .
\end{aligned}
$$

Rearranging Eq. (23), it is obtained that

$$
\begin{aligned}
& s\left[\underline{N}_{1}(t)\right]+u \bar{\lambda}_{1} s\left[\bar{N}_{1}(t)\right](u)=\underline{N}_{1}(0)+r u, \\
& s\left[\bar{N}_{1}(t)\right]+u \underline{\lambda}_{1} s\left[\underline{N}_{1}(t)\right](u)=\bar{N}_{1}(0)+\bar{r} u, \\
& s\left[\underline{N}_{2}(t)\right](u)-u \underline{\lambda}_{1} s\left[\underline{N}_{1}(t)\right](u)+u \bar{\lambda}_{2} s\left[\bar{N}_{2}(t)\right](u)=\underline{N}_{2}(0), \\
& s\left[\bar{N}_{2}(t)\right](u)-u \bar{\lambda}_{1} s\left[\bar{N}_{1}(t)\right](u)+u \underline{\lambda}_{2} s\left[\underline{N}_{2}(t)\right](u)=\bar{N}_{2}(0) .
\end{aligned}
$$

Solving Eq. (24),

$$
\begin{aligned}
s\left[\underline{N}_{1}(t)\right](u)= & \left(-\bar{\lambda}_{1} \bar{N}_{1}(0)+\underline{r}\right) \frac{u}{1-\underline{\lambda}_{1} \bar{\lambda}_{1} u^{2}}-\bar{\lambda}_{1} \bar{r} \frac{u^{2}}{1-\underline{\lambda}_{1} \bar{\lambda}_{1} u^{2}}+\underline{N}_{1}(0) \frac{1}{1-\underline{\lambda}_{1} \bar{\lambda}_{1} u^{2}}, \\
s\left[\bar{N}_{1}(t)\right](u)= & \left(-\underline{\lambda}_{1} \underline{N}_{1}(0)+\bar{r}\right) \frac{u}{1-\underline{\lambda}_{1} \bar{\lambda}_{1} u^{2}}-\underline{\lambda}_{1} \frac{r}{1-\underline{\lambda}_{1} \bar{\lambda}_{1} u^{2}}+\bar{N}_{1}(0) \frac{1}{1-\underline{\lambda}_{1} \bar{\lambda}_{1} u^{2}}, \\
s\left[\underline{N}_{2}(t)\right](u)= & \frac{1}{\left(\underline{\lambda}_{1} \bar{\lambda}_{1} u^{2}-1\right)\left(\underline{\lambda}_{2} \bar{\lambda}_{2} u^{2}-1\right)}\left(\underline{r}_{1} \bar{\lambda}_{1} \bar{\lambda}_{2} u^{4}+\underline{N}_{2}(0)+\left(\underline{\lambda}_{1} \bar{\lambda}_{1} \bar{\lambda}_{2} \bar{N}_{2}(0)+\underline{\lambda}_{1} \bar{\lambda}_{1} \bar{\lambda}_{2} \underline{N}_{1}(0)\right.\right. \\
& \left.-\bar{r}_{1} \bar{\lambda}_{1}-\bar{r} \bar{\lambda}_{2} \bar{\lambda}_{1}\right) u^{3}-\left(\underline{\lambda}_{1} \bar{\lambda}_{1} \underline{N}_{2}(0)-\underline{r} \underline{\lambda}_{1}+\bar{N}_{1}(0) \underline{\lambda}_{1} \bar{\lambda}_{1}+\bar{N}_{1}(0) \bar{\lambda}_{2} \bar{\lambda}_{1}\right) u^{2}-\left(\bar{\lambda}_{2} \bar{N}_{2}(0)\right. \\
& \left.\left.-\underline{N}_{1}(0) \underline{\lambda}_{1}\right) u\right), \\
s\left[\bar{N}_{2}(t)\right](u)= & \frac{1}{\left(\bar{\lambda}_{1} \underline{\lambda}_{1} u^{2}-1\right)\left(\bar{\lambda}_{2} \underline{\lambda}_{2} u^{2}-1\right)}\left(\bar{r} \bar{\lambda}_{1} \underline{\lambda}_{1} \underline{\lambda}_{2} u^{4}+\bar{N}_{2}(0)+\left(\bar{\lambda}_{1} \underline{\lambda}_{1} \underline{\lambda}_{2} \underline{N}_{2}(0)+\bar{\lambda}_{1} \underline{\lambda}_{1} \underline{\lambda}_{2} \bar{N}_{1}(0)\right.\right. \\
& \left.-\underline{r} \bar{\lambda}_{1} \underline{\lambda}_{1}-\underline{r} \underline{\lambda}_{2} \underline{\lambda}_{1}\right) u^{3}-\left(\bar{\lambda}_{1} \underline{\lambda}_{1} \bar{N}_{2}(0)-\bar{r} \bar{\lambda}_{1}+\underline{N}_{1}(0) \bar{\lambda}_{1} \underline{\lambda}_{1}+\underline{N}_{1}(0) \underline{\lambda}_{2} \underline{\lambda}_{1}\right) u^{2}-\left(\underline{\lambda}_{2} \underline{N}_{2}(0)\right. \\
& \left.\left.-\bar{N}_{1}(0) \bar{\lambda}_{1}\right) u\right) .
\end{aligned}
$$

By using the inverse of FST, finally, the solutions are as follows. 


$$
\begin{aligned}
& \underline{N}_{1}(t)=\left(-\bar{\lambda}_{1} \bar{N}_{1}(0)+\underline{r}\right) \frac{1}{\sqrt{\underline{\lambda}_{1} \bar{\lambda}_{1}}} \sinh \left(\sqrt{\underline{\lambda}_{1} \bar{\lambda}_{1}} t\right)+\underline{N}_{1}(0) \cosh \left(\sqrt{\underline{\lambda}_{1} \bar{\lambda}_{1}} t\right)-\bar{\lambda}_{1} \bar{r}\left(\frac{\cosh \left(\sqrt{\underline{\lambda}_{1} \bar{\lambda}_{1}} t\right)-1}{\underline{\lambda}_{1} \bar{\lambda}_{1}}\right), \\
& \bar{N}_{1}(t)=\left(-\underline{\lambda}_{1} \underline{N}_{1}(0)+\bar{r}\right) \frac{1}{\sqrt{\underline{\lambda}_{1} \bar{\lambda}_{1}}} \sinh \left(\sqrt{\underline{\lambda}_{1} \bar{\lambda}_{1}} t\right)+\bar{N}_{1}(0) \cosh \left(\sqrt{\underline{\lambda}_{1} \bar{\lambda}_{1}} t\right)-\underline{\lambda}_{1} \underline{r}\left(\frac{\cosh \left(\sqrt{\underline{\lambda}_{1} \bar{\lambda}_{1}} t\right)-1}{\underline{\lambda}_{1} \bar{\lambda}_{1}}\right), \\
& \underline{N}_{2}(t)=\underline{r \lambda_{1}} \bar{\lambda}_{1} \bar{\lambda}_{2}\left(\frac{e^{-\sqrt{\lambda_{1} \bar{\lambda}_{1}} t}\left(e^{2 \sqrt{\lambda_{1} \bar{\lambda}_{1}} t}+1\right)}{2 \underline{\lambda}_{1} \bar{\lambda}_{1}\left(\underline{\lambda}_{1} \bar{\lambda}_{1}-\underline{\lambda}_{2} \bar{\lambda}_{2}\right)}+\frac{e^{-\sqrt{\lambda_{2} \bar{\lambda}_{2}} t}\left(e^{2 \sqrt{\lambda_{2} \bar{\lambda}_{2}} t}+1\right)}{2 \underline{\lambda}_{2} \bar{\lambda}_{2}\left(\underline{\lambda}_{2} \bar{\lambda}_{2}-\underline{\lambda}_{1} \bar{\lambda}_{1}\right)}+\frac{1}{\underline{\lambda}_{1} \bar{\lambda}_{1} \underline{\lambda}_{2} \bar{\lambda}_{2}}\right)+\underline{N}_{2}(0)
\end{aligned}
$$

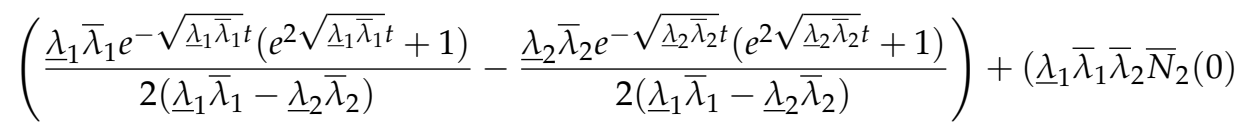

$$
\begin{aligned}
& \left.+\underline{\lambda}_{1} \bar{\lambda}_{1} \bar{\lambda}_{2} \underline{N}_{1}(0)-\bar{r} \underline{\lambda}_{1} \bar{\lambda}_{1}-\bar{r} \bar{\lambda}_{2} \bar{\lambda}_{1}\right)\left(\frac{e^{-\sqrt{\lambda_{1} \bar{\lambda}_{1}} t}\left(e^{2 \sqrt{\lambda_{1} \bar{\lambda}_{1}} t}-1\right)}{2 \sqrt{\underline{\lambda}_{1} \bar{\lambda}_{1}}\left(\underline{\lambda}_{1} \bar{\lambda}_{1}-\underline{\lambda}_{2} \bar{\lambda}_{2}\right)}+\frac{e^{-\sqrt{\underline{\lambda}_{2} \bar{\lambda}_{2}} t}\left(e^{2 \sqrt{\lambda_{2} \bar{\lambda}_{2}} t}-1\right)}{2 \sqrt{\underline{\lambda}_{2} \bar{\lambda}_{2}}\left(\underline{\lambda}_{2} \bar{\lambda}_{2}-\underline{\lambda}_{1} \bar{\lambda}_{1}\right)}\right) \\
& -\left(\underline{\lambda}_{1} \bar{\lambda}_{1} \underline{N}_{2}(0)-\underline{r} \underline{\lambda}_{1}+\bar{N}_{1}(0) \underline{\lambda}_{1} \bar{\lambda}_{1}+\bar{N}_{1}(0) \bar{\lambda}_{2} \bar{\lambda}_{1}\right) \\
& \left(\frac{e^{-\sqrt{\lambda_{1} \bar{\lambda}_{1}} t}\left(e^{2 \sqrt{\lambda_{1} \bar{\lambda}_{1}} t}+1\right)}{2\left(\underline{\lambda}_{1} \bar{\lambda}_{1}-\underline{\lambda}_{2} \bar{\lambda}_{2}\right)}-\frac{e^{-\sqrt{\lambda_{2} \bar{\lambda}_{2}} t}\left(e^{2 \sqrt{\lambda_{2} \bar{\lambda}_{2}} t}+1\right)}{2\left(\underline{\lambda}_{1} \bar{\lambda}_{1}-\underline{\lambda}_{2} \bar{\lambda}_{2}\right)}\right) \\
& -\left(\bar{\lambda}_{2} \bar{N}_{2}(0)-\underline{N}_{1}(0) \underline{\lambda}_{1}\right)\left(\frac{\sqrt{\underline{\lambda}_{1} \bar{\lambda}_{1}} e^{-\sqrt{\underline{\lambda}_{1} \bar{\lambda}_{1}} t}\left(e^{2 \sqrt{\underline{\lambda}_{1} \bar{\lambda}_{1}} t}-1\right)}{2\left(\underline{\lambda}_{1} \bar{\lambda}_{1}-\underline{\lambda}_{2} \bar{\lambda}_{2}\right)}-\frac{\sqrt{\underline{\lambda}_{2} \bar{\lambda}_{2}} e^{-\sqrt{\underline{\lambda}_{2} \bar{\lambda}_{2}} t}\left(e^{2 \sqrt{\underline{\lambda}_{2} \bar{\lambda}_{2}} t}-1\right)}{2\left(\underline{\lambda}_{1} \bar{\lambda}_{1}-\underline{\lambda}_{2} \bar{\lambda}_{2}\right)}\right), \\
& \bar{N}_{2}(t)=\bar{r} \underline{\lambda}_{1} \bar{\lambda}_{1} \underline{\lambda}_{2}\left(\frac{e^{-\sqrt{\lambda_{1} \bar{\lambda}_{1}} t}\left(e^{2 \sqrt{\lambda_{1} \bar{\lambda}_{1}} t}+1\right)}{2 \underline{\lambda}_{1} \bar{\lambda}_{1}\left(\underline{\lambda}_{1} \bar{\lambda}_{1}-\underline{\lambda}_{2} \bar{\lambda}_{2}\right)}+\frac{e^{-\sqrt{\lambda_{2} \bar{\lambda}_{2}} t}\left(e^{2 \sqrt{\lambda_{2} \bar{\lambda}_{2}} t}+1\right)}{2 \underline{\lambda}_{2} \bar{\lambda}_{2}\left(\underline{\lambda}_{2} \bar{\lambda}_{2}-\underline{\lambda}_{1} \bar{\lambda}_{1}\right)}+\frac{1}{\underline{\lambda}_{1} \bar{\lambda}_{1} \underline{\lambda}_{2} \bar{\lambda}_{2}}\right)+\bar{N}_{2}(0) \\
& \left(\frac{\underline{\lambda}_{1} \bar{\lambda}_{1} e^{-\sqrt{\lambda_{1} \bar{\lambda}_{1}} t}\left(e^{2 \sqrt{\lambda_{1} \bar{\lambda}_{1}} t}+1\right)}{2\left(\underline{\lambda}_{1} \bar{\lambda}_{1}-\underline{\lambda}_{2} \bar{\lambda}_{2}\right)}-\frac{\underline{\lambda}_{2} \bar{\lambda}_{2} e^{-\sqrt{\lambda_{2} \bar{\lambda}_{2}} t}\left(e^{2 \sqrt{\underline{\lambda}_{2} \bar{\lambda}_{2}} t}+1\right)}{2\left(\underline{\lambda}_{1} \bar{\lambda}_{1}-\underline{\lambda}_{2} \bar{\lambda}_{2}\right)}\right)+\left(\underline{\lambda}_{1} \bar{\lambda}_{1} \underline{\lambda}_{2} \underline{N}_{2}(0)\right. \\
& \left.+\underline{\lambda}_{1} \bar{\lambda}_{1} \underline{\lambda}_{2} \bar{N}_{1}(0)-\underline{r} \underline{\lambda}_{1} \bar{\lambda}_{1}-\underline{r} \underline{\lambda}_{2} \underline{\lambda}_{1}\right)\left(\frac{e^{-\sqrt{\underline{\lambda}_{1} \bar{\lambda}_{1}} t}\left(e^{2 \sqrt{\underline{\lambda}_{1} \bar{\lambda}_{1}} t}-1\right)}{2 \sqrt{\underline{\lambda}_{1} \bar{\lambda}_{1}}\left(\underline{\lambda}_{1} \bar{\lambda}_{1}-\underline{\lambda}_{2} \bar{\lambda}_{2}\right)}+\frac{e^{-\sqrt{\underline{\lambda}_{2} \bar{\lambda}_{2}} t}\left(e^{2 \sqrt{\underline{\lambda}_{2} \bar{\lambda}_{2}} t}-1\right)}{2 \sqrt{\underline{\lambda}_{2} \bar{\lambda}_{2}}\left(\underline{\lambda}_{2} \bar{\lambda}_{2}-\underline{\lambda}_{1} \bar{\lambda}_{1}\right)}\right) \\
& -\left(\underline{\lambda}_{1} \bar{\lambda}_{1} \bar{N}_{2}(0)-\bar{r} \bar{\lambda}_{1}+\underline{N}_{1}(0) \underline{\lambda}_{1} \bar{\lambda}_{1}+\underline{N}_{1}(0) \underline{\lambda}_{2} \underline{\lambda}_{1}\right) \\
& \left(\frac{e^{-\sqrt{\lambda_{1} \bar{\lambda}_{1}} t}\left(e^{2 \sqrt{\lambda_{1} \bar{\lambda}_{1}} t}+1\right)}{2\left(\underline{\lambda}_{1} \bar{\lambda}_{1}-\underline{\lambda}_{2} \bar{\lambda}_{2}\right)}-\frac{e^{-\sqrt{\lambda_{2} \bar{\lambda}_{2}} t}\left(e^{2 \sqrt{\lambda_{2} \bar{\lambda}_{2}} t}+1\right)}{2\left(\underline{\lambda}_{1} \bar{\lambda}_{1}-\underline{\lambda}_{2} \bar{\lambda}_{2}\right)}\right) \\
& -\left(\underline{\lambda}_{2} \underline{N}_{2}(0)-\bar{N}_{1}(0) \bar{\lambda}_{1}\right)\left(\frac{\sqrt{\underline{\lambda}_{1} \bar{\lambda}_{1}} e^{-\sqrt{\lambda_{1} \bar{\lambda}_{1}} t}\left(e^{2 \sqrt{\underline{\lambda}_{1} \bar{\lambda}_{1}} t}-1\right)}{2\left(\underline{\lambda}_{1} \bar{\lambda}_{1}-\underline{\lambda}_{2} \bar{\lambda}_{2}\right)}-\frac{\sqrt{\underline{\lambda}_{2} \bar{\lambda}_{2}} e^{-\sqrt{\lambda_{2} \bar{\lambda}_{2}} t}\left(e^{2 \sqrt{\underline{\lambda}_{2} \bar{\lambda}_{2}} t}-1\right)}{2\left(\underline{\lambda}_{1} \bar{\lambda}_{1}-\underline{\lambda}_{2} \bar{\lambda}_{2}\right)}\right) .
\end{aligned}
$$

Remark 1. The results in Case 1 are similar to results obtained by the authors in [19] and [33]. However, the authors in [19] and [33] only considered the condition when both $N_{1}(t)$ and $N_{2}(t)$ are (i)-differentiable. To the best of our knowledge, this is the first time the solution for Case $2\left(N_{1}(t)\right.$ and $N_{2}(t)$ to be (ii)-differentiable) for problem adapted from fuzzy radioactive decay problem is provided in details. 
Case 2: Next, consider that both $N_{1}(t)$ and $N_{2}(t)$ to be (ii)-differentiable. So,

$$
\begin{aligned}
& \frac{s\left[\underline{N}_{1}(t)\right]-\underline{N}_{1}(0)}{u}=-\underline{\lambda}_{1} s\left[\underline{N}_{1}(t)\right]+\bar{r}, \\
& \frac{s\left[\bar{N}_{1}(t)\right]-\bar{N}_{1}(0)}{u}=-\bar{\lambda}_{1} s\left[\bar{N}_{1}(t)\right]+\underline{r}, \\
& \frac{s\left[\underline{N}_{2}(t)\right]-\underline{N}_{2}(0)}{u}=\bar{\lambda}_{1} s\left[\bar{N}_{1}(t)\right]-\underline{\lambda}_{2} s\left[\underline{N}_{2}(t)\right], \\
& \frac{s\left[\bar{N}_{2}(t)\right]-\bar{N}_{2}(0)}{u}=\underline{\lambda}_{1} s\left[\underline{N}_{1}(t)\right]-\bar{\lambda}_{2} s\left[\bar{N}_{2}(t)\right] .
\end{aligned}
$$

Rearranging Eq. (25),

$$
\begin{aligned}
& \left(1+\underline{\lambda}_{1} u\right) s\left[\underline{N}_{1}(t)\right]=\underline{N}_{1}(0)+\bar{r} u, \\
& \left(1+\bar{\lambda}_{1} u\right) s\left[\bar{N}_{1}(t)\right]=\bar{N}_{1}(0)+\underline{r} u, \\
& \left(1+\underline{\lambda}_{2} u\right) s\left[\underline{N}_{2}(t)\right]-\bar{\lambda}_{1} s\left[\bar{N}_{1}(t)\right] u=\underline{N}_{2}(0), \\
& \left(1+\bar{\lambda}_{2} u\right) s\left[\bar{N}_{2}(t)\right]-\underline{\lambda}_{1} s\left[\underline{N}_{1}(t)\right] u=\bar{N}_{2}(0) .
\end{aligned}
$$

Solving Eq. (26),

$$
\begin{aligned}
& s\left[\underline{N}_{1}(t)\right]=\underline{N}_{1}(0) \frac{1}{\left(1+\underline{\lambda}_{1} u\right)}+\bar{r} \frac{u}{\left(1+\underline{\lambda}_{1} u\right)}, \\
& s\left[\bar{N}_{1}(t)\right]=\bar{N}_{1}(0) \frac{1}{\left(1+\bar{\lambda}_{1} u\right)}+\underline{r} \frac{u}{\left(1+\bar{\lambda}_{1} u\right)}, \\
& s\left[\underline{N}_{2}(t)\right]=\underline{r} \bar{\lambda}_{1} \frac{u^{2}}{\left(1+u \bar{\lambda}_{1}\right)\left(1+u \underline{\lambda}_{2}\right)}+\underline{N}_{2}(0) \frac{1}{1+u \underline{\lambda}_{2}}+\bar{N}_{1}(0) \bar{\lambda}_{1} \frac{u}{\left(1+u \bar{\lambda}_{1}\right)\left(1+u \underline{\lambda}_{2}\right)}, \\
& s\left[\bar{N}_{2}(t)\right]=\bar{r}_{1} \frac{u^{2}}{\left(1+u \underline{\lambda}_{1}\right)\left(1+u \bar{\lambda}_{2}\right)}+\bar{N}_{2}(0) \frac{1}{1+u \bar{\lambda}_{2}}+\underline{N}_{1}(0) \bar{\lambda}_{1} \frac{u}{\left(1+u \underline{\lambda}_{1}\right)\left(1+u \bar{\lambda}_{2}\right)} .
\end{aligned}
$$

By using the inverse of FST, the solutions obtained are as follows.

$$
\begin{aligned}
& \underline{N}_{1}(t)=\underline{N}_{1}(0) e^{-\underline{\lambda}_{1} t}+\bar{r} \frac{1-e^{-\underline{\lambda}_{1} t}}{\underline{\lambda}_{1}} \\
& \bar{N}_{1}(t)=\bar{N}_{1}(0) e^{-\bar{\lambda}_{1} t}+\underline{r} \frac{1-e^{-\bar{\lambda}_{1} t}}{\bar{\lambda}_{1}} \\
& \underline{N}_{2}(t)=\underline{r} \bar{\lambda}_{1} \frac{1}{\underline{\lambda}_{2}-\bar{\lambda}_{1}}\left(\frac{1-e^{-\bar{\lambda}_{1} t}}{\bar{\lambda}_{1}}-\frac{1-e^{-\underline{\lambda}_{2} t}}{\underline{\lambda}_{2}}\right)+\bar{N}_{1}(0) \bar{\lambda}_{1} \frac{1}{\underline{\lambda}_{2}-\bar{\lambda}_{1}}\left(e^{-\bar{\lambda}_{1} t}-e^{-\underline{\lambda}_{2} t}\right)+\underline{N}_{2}(0) e^{-\underline{\lambda}_{2} t}, \\
& \bar{N}_{2}(t)=\underline{\bar{r}}_{1} \frac{1}{\bar{\lambda}_{2}-\underline{\lambda}_{1}}\left(\frac{1-e^{-\underline{\lambda}_{1} t}}{\underline{\lambda}_{1}}-\frac{1-e^{-\bar{\lambda}_{2} t}}{\bar{\lambda}_{2}}\right)+\underline{N}_{1}(0) \underline{\lambda}_{1} \frac{1}{\bar{\lambda}_{2}-\underline{\lambda}_{1}}\left(e^{-\underline{\lambda}_{1} t}-e^{-\bar{\lambda}_{2} t}\right)+\bar{N}_{2}(0) e^{-\bar{\lambda}_{2} t}
\end{aligned}
$$

For illustration in tables and graphs, we let $r=(4.9,5,5.1), N_{1}=(995,1000,1005), N_{2}=(0,0,0)$, $\widetilde{\lambda}_{1}=(0.2,0.3,0.4)$ and $\widetilde{\lambda}_{2}=(0.02,0.03,0.04)$ respectively. Their parametric representations can be calculated as in Definition 1. The results of $N_{1}(t)$ and $N_{2}(t)$ for Case 1 are illustrated in Figs. 1 and 2, plotted using MATLAB 2017 software, and the values are listed in Tables 1 and 2. The results of $N_{1}(t)$ and $N_{2}(t)$ for Case 2 are illustrated in Figs. 3 and 4, also using MATLAB 2017 software. The numerical values corresponding to this case are in Tables 3 and 4.

It can be seen that for Case 1, the results expand as t increases. This is in accord with the concept of fuzzy derivatives for (i)-differentiability when using the strongly generalized differentiability to interpret FDEs. While for Case 2, the results implicate that the solutions contract as the values of $t$ increases. Similarly, this condition is in accord with the concept of fuzzy derivatives for (ii)-differentiability. 
For the sake of comparison, we would like to emphasized that the results obtained when $\alpha=1$ are similar to the solutions for system of ordinary differential equations. Thus, it can be concluded that SLFDE is an extension of the system of ordinary differential equations. Furthermore, we can also said that SLFDE is a generalized version of system of ordinary differential equations since the set of real numbers $\mathbb{R}$ are subset of the set of fuzzy numbers or fuzzy real numbers $\mathcal{F}(\mathbb{R})$ The advantage of the solutions that we provided in this paper, is that this system are able to cope with uncertainties at initial values as well as having FCCs. This is very common when dealing with real life problems.

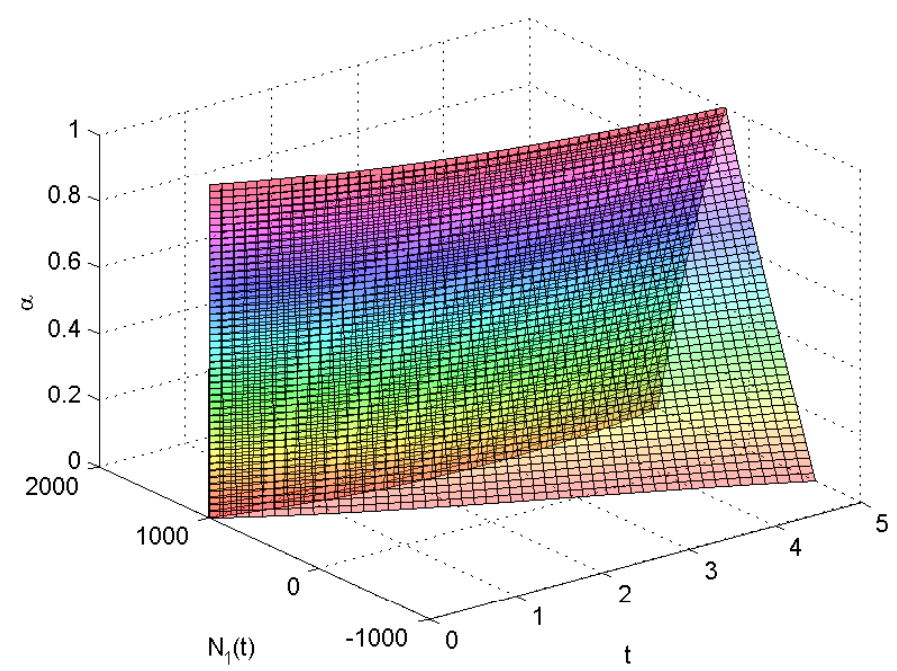

Figure 1. The graphical solution of $N_{1}(t)$ in Eq. (20) for Case 1.

\section{Conclusions}

In this work, we have studied FST for solving SLFDEs with FCCs. One of the advantage of FST, the scale preserving property has been highlighted in Subsection 3.1. This is done to justify why we chose to utilize FST in this paper. Then, procedures for obtaining the solutions of SLFDEs using FST are constructed in Section 5. The process for finding the solutions is then demonstrated on a numerical

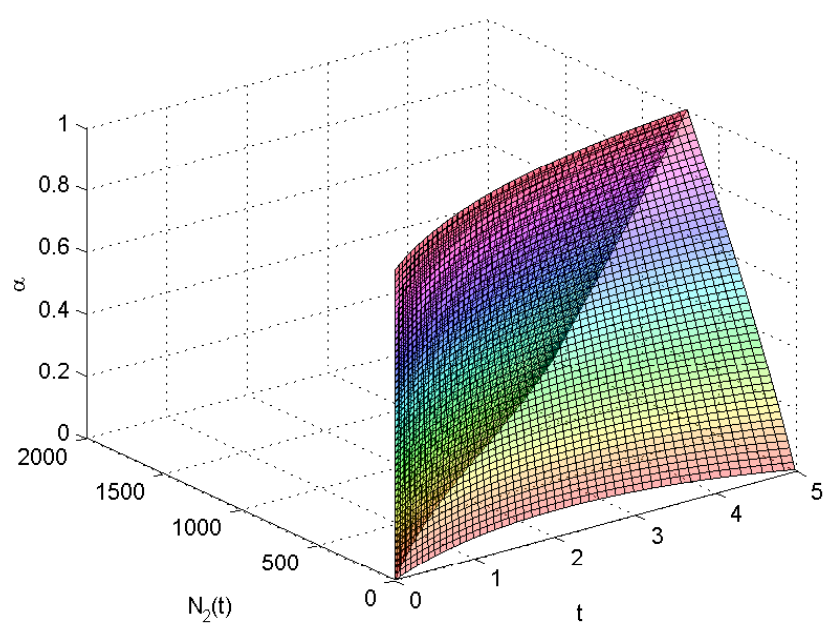

Figure 2. The graphical solution of $N_{2}(t)$ in Eq. (20) for Case 1. 


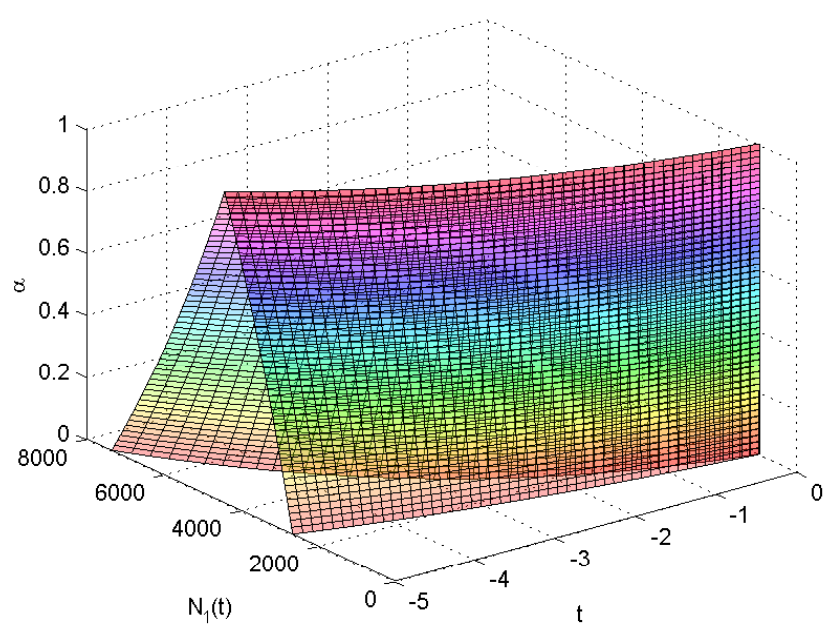

Figure 3. The graphical solution of $N_{1}(t)$ in Eq. (20) for Case 2.

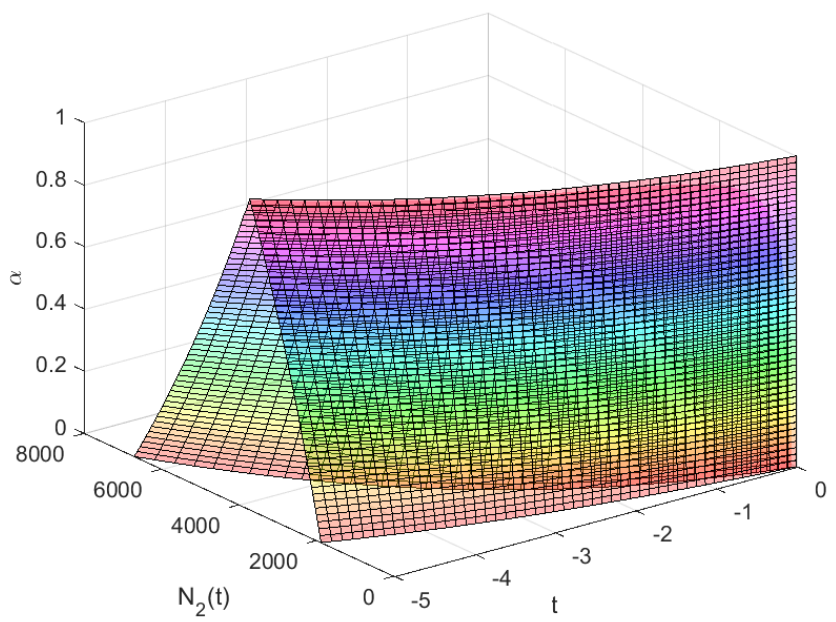

Figure 4. The graphical solution of $N_{2}(t)$ in Eq. (20) for Case 2.

Table 1. The solutions of $N_{1}(t)$ in Eq. (20) for Case 1.

\begin{tabular}{lllllll}
\hline \multirow{2}{*}{$\alpha$} & \multicolumn{2}{c}{$t=0.00$} & \multicolumn{2}{c}{$t=2.00$} & \multicolumn{2}{c}{$t=4.00$} \\
\cline { 2 - 7 } & $\underline{N}_{1}(t)$ & $\bar{N}_{1}(t)$ & $\underline{N}_{1}(t)$ & $\bar{N}_{1}(t)$ & $\underline{N}_{1}(t)$ & $\bar{N}_{1}(t)$ \\
\hline 0.0 & 995.0000 & 1005.0000 & 317.0607 & 759.3051 & -265.0646 & 759.1110 \\
0.1 & 995.5000 & 1004.5000 & 342.3528 & 740.8885 & -203.7037 & 722.4528 \\
0.2 & 996.0000 & 1004.0000 & 367.3685 & 722.0330 & -142.8925 & 683.8660 \\
0.3 & 996.5000 & 1003.5000 & 392.0974 & 702.7460 & -82.7248 & 643.4041 \\
0.4 & 997.0000 & 1003.0000 & 416.5293 & 683.0353 & -23.2936 & 601.1243 \\
0.5 & 997.5000 & 1002.5000 & 440.6543 & 662.9089 & 35.3101 & 557.0864 \\
0.6 & 998.0000 & 1002.0000 & 464.4623 & 642.3748 & 92.9964 & 511.3538 \\
0.7 & 998.5000 & 1001.5000 & 487.9435 & 621.4415 & 149.6776 & 463.9925 \\
0.8 & 999.0000 & 1001.0000 & 511.0884 & 600.1174 & 205.2677 & 415.0716 \\
0.9 & 999.5000 & 1000.5000 & 533.8875 & 578.4111 & 259.6827 & 364.6629 \\
1.0 & 1000.0000 & 1000.0000 & 556.3314 & 556.3314 & 312.8410 & 312.8410 \\
\hline
\end{tabular}


Table 2. The solutions of $N_{2}(t)$ in Eq. (20) for Case 1.

\begin{tabular}{cccllll}
\hline \multirow{2}{*}{$\alpha$} & \multicolumn{2}{c}{$t=0.00$} & \multicolumn{2}{c}{$t=2.00$} & \multicolumn{2}{c}{$t=4.00$} \\
\cline { 2 - 7 } & $\underline{N}_{2}(t)$ & $\bar{N}_{2}(t)$ & $\underline{N}_{2}(t)$ & $\bar{N}_{2}(t)$ & $\underline{N}_{2}(t)$ & $\bar{N}_{2}(t)$ \\
\hline 0.0 & 0.0000 & 0.0000 & 227.2468 & 682.1144 & 159.7583 & 1265.3728 \\
0.1 & 0.0000 & 0.0000 & 246.7859 & 656.6515 & 202.8278 & 1202.1398 \\
0.2 & 0.0000 & 0.0000 & 266.7095 & 631.4204 & 247.5739 & 1139.2478 \\
0.3 & 0.0000 & 0.0000 & 287.0105 & 606.4306 & 293.9422 & 1076.7858 \\
0.4 & 0.0000 & 0.0000 & 307.6815 & 581.6917 & 341.8578 & 1014.8416 \\
0.5 & 0.0000 & 0.0000 & 328.7148 & 557.2130 & 391.3146 & 953.5020 \\
0.6 & 0.0000 & 0.0000 & 350.1029 & 533.0038 & 442.1961 & 892.8524 \\
0.7 & 0.0000 & 0.0000 & 371.8379 & 509.0733 & 494.4550 & 832.9766 \\
0.8 & 0.0000 & 0.0000 & 393.9118 & 485.4305 & 548.0236 & 773.9570 \\
0.9 & 0.0000 & 0.0000 & 416.3164 & 462.0842 & 602.8317 & 715.8741 \\
1.0 & 0.0000 & 0.0000 & 439.0433 & 439.0433 & 658.8067 & 658.8067 \\
\hline
\end{tabular}

Table 3. The solutions of $N_{1}(t)$ in Eq. (20) for Case 2.

\begin{tabular}{lllllll}
\hline \multirow{2}{*}{$\alpha$} & \multicolumn{2}{c}{$t=-4.00$} & \multicolumn{2}{c}{$t=-2.00$} & \multicolumn{2}{c}{$t=0.00$} \\
\cline { 2 - 7 } & $\underline{N}_{1}(t)$ & $\bar{N}_{1}(t)$ & $\underline{N}_{1}(t)$ & $\bar{N}_{1}(t)$ & $\underline{N}_{1}(t)$ & $\bar{N}_{1}(t)$ \\
\hline 0.0 & 2183.2000 & 4929.4000 & 1471.8000 & 2221.7000 & 995.0000 & 1005.0000 \\
0.1 & 2274.0000 & 4732.9000 & 1502.5000 & 2176.4000 & 995.5000 & 1004.5000 \\
0.2 & 2368.7000 & 4544.3000 & 1533.7000 & 2132.1000 & 996.0000 & 1004.0000 \\
0.3 & 2467.2000 & 4363.1000 & 1565.7000 & 2088.7000 & 996.5000 & 1003.5000 \\
0.4 & 2569.9000 & 4189.2000 & 1598.2000 & 2046.1000 & 997.0000 & 1003.0000 \\
0.5 & 2676.8000 & 4022.1000 & 1631.5000 & 2004.4000 & 997.5000 & 1002.5000 \\
0.6 & 2788.1000 & 3861.7000 & 1665.4000 & 1963.6000 & 998.0000 & 1002.0000 \\
0.7 & 2904.0000 & 3707.7000 & 1700.1000 & 1923.6000 & 998.5000 & 1001.5000 \\
0.8 & 3024.8000 & 3559.8000 & 1735.5000 & 1884.4000 & 999.0000 & 1001.0000 \\
0.9 & 3150.5000 & 3417.8000 & 1771.6000 & 1846.0000 & 999.5000 & 1000.5000 \\
1.0 & 3281.4000 & 3281.4000 & 1808.4000 & 1808.4000 & 1000.0000 & 1000.0000 \\
\hline
\end{tabular}

Table 4. The solutions of $N_{2}(t)$ in Eq. (20) for Case 2.

\begin{tabular}{lllllll}
\hline \multirow{2}{*}{$\alpha$} & \multicolumn{2}{c}{$t=-4.00$} & \multicolumn{2}{c}{$t=2.00$} & \multicolumn{2}{c}{$t=0.00$} \\
\cline { 2 - 7 } & $\underline{N}_{2}(t)$ & $\bar{N}_{2}(t)$ & $\underline{N}_{2}(t)$ & $\bar{N}_{2}(t)$ & $\underline{N}_{2}(t)$ & $\bar{N}_{2}(t)$ \\
\hline 0.0 & 4123.2773 & 1319.8732 & 1258.5979 & 510.5217 & 0.0000 & 0.0000 \\
0.1 & 3925.9438 & 1415.0427 & 1213.9111 & 541.4794 & 0.0000 & 0.0000 \\
0.2 & 3736.1399 & 1513.8730 & 1170.0869 & 573.0349 & 0.0000 & 0.0000 \\
0.3 & 3553.5696 & 1616.5135 & 1127.1079 & 605.2005 & 0.0000 & 0.0000 \\
0.4 & 3377.9487 & 1723.1200 & 1084.9570 & 637.9888 & 0.0000 & 0.0000 \\
0.5 & 3209.0041 & 1833.8546 & 1043.6176 & 671.4126 & 0.0000 & 0.0000 \\
0.6 & 3046.4736 & 1948.8858 & 1003.0733 & 705.4850 & 0.0000 & 0.0000 \\
0.7 & 2890.1052 & 2068.3893 & 963.3081 & 740.2193 & 0.0000 & 0.0000 \\
0.8 & 2739.6568 & 2192.5479 & 924.3062 & 775.6291 & 0.0000 & 0.0000 \\
0.9 & 2594.8959 & 2321.5519 & 886.0523 & 811.7284 & 0.0000 & 0.0000 \\
1.0 & 2455.5991 & 2455.5991 & 848.5313 & 848.5313 & 0.0000 & 0.0000 \\
\hline
\end{tabular}

example adapted from fuzzy radioactive decay model and this can be seen in Section 6 . We believe that this research will be beneficial for scientists and engineers when they face problems that are 
surrounded with fuzziness. For future research, we intend to explore other real life problems. Besides, we will also focus on integrating FST for solving system of fuzzy nonlinear problems.

\section{Conflict of Interests}

The authors declared that there is no conflict of interest.

Author Contributions: Conceptualization, N.A. Abdul Rahman and M.Z. Ahmad; Methodology, N.A. Abdul Rahman and M.Z. Ahmad; Validation, N.A. Abdul Rahman and M.Z. Ahmad; Writing-Original Draft Preparation, N.A. Abdul Rahman; Writing-Review \& Editing,N.A. Abdul Rahman and M.Z. Ahmad; Visualization, N.A. Abdul Rahman

Acknowledgments: We would like to thank Universiti Sains Malaysia and Universiti Malaysia Perlis for providing facilities throughout this research.

Conflicts of Interest: The authors declare no conflict of interest.

\section{Abbreviations}

The following abbreviations are used in this manuscript:

FDE Fuzzy differential equation

FST Fuzzy Sumudu transform

SLFDE System of linear fuzzy differential equation

FCC Fuzzy constant coefficient

\section{References}

1. Guo, Y.; Wang, Y. Decay of dissipative equations and negative Sobolev spaces. Communications in Partial Differential Equations 2012, 37, 2165-2208.

2. Wang, M.; Zhang, Y. Two kinds of free boundary problems for the diffusive prey-predator model. Nonlinear Analysis: Real World Applications 2015, 24, 73-82.

3. Momani, S.; Odibat, Z. Numerical approach to differential equations of fractional order. Journal of Computational and Applied Mathematics 2007, 207, 96 - 110.

4. Caraballo, T.; Cheban, D. Almost periodic and almost automorphic solutions of linear differential/difference equations without Favard's separation condition. I. Journal of Differential Equations 2009, 246, $108-128$.

5. Chirkunov, Y.A. Linear autonomy conditions for the basic Lie algebra of a system of linear differential equations. Doklady Mathematics 2009, 79, 415-417.

6. Zadeh, L.A. Fuzzy sets. Information and control 1965, 8, 338-353.

7. Chang, S.S.; Zadeh, L.A. On fuzzy mapping and control. IEEE Transactions on Systems, Man, and Cybernetics 1972, pp. 30-34.

8. $\quad \mathrm{Xu}$, J.; Liao, Z.; Hu, Z. A class of linear differential dynamical systems with fuzzy initial condition. Fuzzy Sets and Systems 2007, 158, 2339-2358.

9. Puri, M.L.; Ralescu, D.A. Differentials of fuzzy functions. Journal of Mathematical Analysis and Applications 1983, 91, 552-558.

10. Ma, M.; Friedman, M.; Kandel, A. Numerical solutions of fuzzy differential equations. Fuzzy Sets and Systems 1999, 105, 133-138.

11. Chalco-Cano, Y.; Román-Flores, H. On new solutions of fuzzy differential equations. Chaos, Solitons $\mathcal{E}$ Fractals 2008, 38, 112-119.

12. Bede, B.; Rudas, I.J.; Bencsik, A.L. First order linear fuzzy differential equations under generalized differentiability. Information Sciences 2007, 177, 1648-1662.

13. Ahmad, M.Z.; Abdul Rahman, N.A. Explicit solution of fuzzy differential equations by mean of fuzzy Sumudu transform. International Journal of Applied Physics and Mathematics 2015, 5, 86-93.

14. Abdul Rahman, N.A.; Ahmad, M.Z. Applications of the fuzzy Sumudu transform for the solution of first order fuzzy differential equations. Entropy 2015, 17, 4582-4601.

15. Abdul Rahman, N.A.; Ahmad, M.Z. Fuzzy Sumudu transform for solving fuzzy partial differential equations. The Journal of Nonlinear Science and Applications 2016, 9, 3226-3239. 
16. Haydar, A.K. Fuzzy Sumudu transform for fuzzy nth-order derivative and solving fuzzy ordinary differential equations. International Journal of Science and Research 2015, 4, 1372-1378.

17. Abdul Rahman, N.A.; Ahmad, M.Z. Solving Fuzzy Fractional Differential Equations using Fuzzy Sumudu Transform. The Journal of Nonlinear Science and Applications 2017, 6, 19-28.

18. Abdul Rahman, N.A.; Ahmad, M.Z. Solving Fuzzy Volterra Integral Equations via Fuzzy Sumudu Transform. Applied Mathematics and Computational Intelligence 2017, 10, 2620-2632.

19. Fard, O.S.; Ghal-Eh, N. Numerical solutions for linear system of first-order fuzzy differential equations with fuzzy constant coefficients. Information Sciences 2011, 181, $4765-4779$.

20. Gouyandeh, Z.; Armand, A. Numerical solutions of fuzzy linear system differential equations and application of a radioactivity decay model. Communications on Advanced Computational Science with Applications 2013, 2013, 1-11.

21. Allahviranloo, T. The Adomian decomposition method for fuzzy system of linear equations. Applied Mathematics and Computation 2005, 163, 553 - 563.

22. Khastan, A.; Nieto, J.J.; Rodríguez-López, R. Periodic boundary value problems for first-order linear differential equations with uncertainty under generalized differentiability. Information Sciences 2013, 222, 544-558.

23. Mosleh, M. Fuzzy neural network for solving a system of fuzzy differential equations. Applied Soft Computing 2013, 13, 3597-3607.

24. Mosleh, M.; Otadi, M. Approximate solution of fuzzy differential equations under generalized differentiability. Applied Mathematical Modelling 2015, 39, 3003-3015.

25. Allahviranloo, T.; Ahmadi, M.B. Fuzzy Laplace transforms. Soft Computing 2010, 14, $235-243$.

26. Kaleva, O. A note on fuzzy differential equations. Nonlinear Analysis: Theory, Methods E Applications 2006, 64, 895-900.

27. Friedman, M.; Ma, M.; Kandel, A. Numerical solutions of fuzzy differential and integral equations. Fuzzy Sets and Systems 1999, 106, 35-48.

28. Kaufmann, A.; Gupta, M.M. Introduction to fuzzy arithmetic: Theory and applications; Van Nostrand Reinhold: New York, 1985.

29. Wu, H.C. The improper fuzzy Riemann integral and its numerical integration. Information Sciences 1998, 111, 109-137.

30. Bede, B.; Gal, S.G. Generalizations of the differentiability of fuzzy-number-valued functions with applications to fuzzy differential equations. Fuzzy Sets and Systems 2005, 151, 581-599.

31. Guang-Quan, Z. Fuzzy continuous function and its properties. Fuzzy Sets and Systems 1991, 43, 159 - 171.

32. Allahviranloo, T.; Ahmady, N.; Ahmady, E. Numerical solution of fuzzy differential equations by predictor-corrector method. Information sciences 2007, 177, 1633-1647.

33. Najariyan, M.; Mazandarani, M. A note on Numerical solutions for linear system of first-order fuzzy differential equations with fuzzy constant coefficients. Information Sciences 2015, 305, 93 - 96. 